\title{
Effects of Recombinant Human Angiotensin- Converting Enzyme 2 on Response to Acute Hypoxia and Exercise: A Randomised, Placebo-Controlled Study
}

\author{
David A. Hall (D) · Kate Hanrott · Philipp Badorrek • Dominik Berliner • \\ David C. Budd · Rhena Eames · William M. Powley · Deborah Hewens • \\ Sarah Siederer · Aili L. Lazaar · Anthony Cahn · Jens M. Hohlfeld
}

Received: April 15, 2021 / Accepted: June 14, 2021 / Published online: June 26, 2021

(C) The Author(s) 2021

\begin{abstract}
Introduction: Angiotensin-converting enzyme 2 (ACE2) is a key enzyme of the renin-angiotensin system (RAS) that has been implicated in the pathogenesis of acute respiratory distress syndrome (ARDS). Enhancing ACE2 activity using GSK2586881, a recombinant form of human ACE2, could be beneficial in diseases such as ARDS but may blunt the hypoxic pulmonary vasoconstriction (HPV) response and potentially impact systemic and tissue oxygenation. This study aimed to evaluate the effect of GSK2586881 $0.8 \mathrm{mg} / \mathrm{kg}$ on HPV response in healthy adult volunteers during exercise under hypoxic conditions.
\end{abstract}

Supplementary Information The online version contains supplementary material available at https:// doi.org/10.1007/s41030-021-00164-7.

D. A. Hall $(\bowtie) \cdot$ K. Hanrott · D. C. Budd .

R. Eames · W. M. Powley · D. Hewens · A. Cahn Novel Human Genetics Research Unit, Medicines Research Centre, GlaxoSmithKline Plc., Gunnels Wood Road, Stevenage SG1 2NY, Hertfordshire, UK e-mail: david.a.hall@gsk.com

P. Badorrek · J. M. Hohlfeld

Department of Clinical Airway Research, Fraunhofer Institute of Toxicology and Experimental Medicine, Hannover, Germany

D. Berliner

Department of Cardiology and Angiology,

Hannover Medical School, Hannover, Germany
Methods: In this phase I, randomised, doubleblind (sponsor open) study, GSK2586881 or placebo was administered as a single intravenous (IV) dose in a two-period crossover design. Treatment periods were separated by a washout period of 3-14 days. The primary endpoint was change from baseline in pulmonary artery systolic pressure (PASP) measured by echocardiography. Secondary endpoints included RAS peptides and oxygen saturation.

Results: Seventeen adults aged 18-40 years were randomised to treatment. There were no clinically relevant differences (defined as a reduction of $\geq 5 \mathrm{mmHg}$ ) in change from baseline in PASP between GSK2586881 and placebo. GSK2586881 was well tolerated, with no serious adverse events, no worsening of hypoxaemia and no evidence of immunogenicity. The study was terminated early after review of the data, which showed that the predefined success criteria had not been met.

S. Siederer

Clinical Pharmacology Modelling and Simulation, GlaxoSmithKline, Stevenage, UK

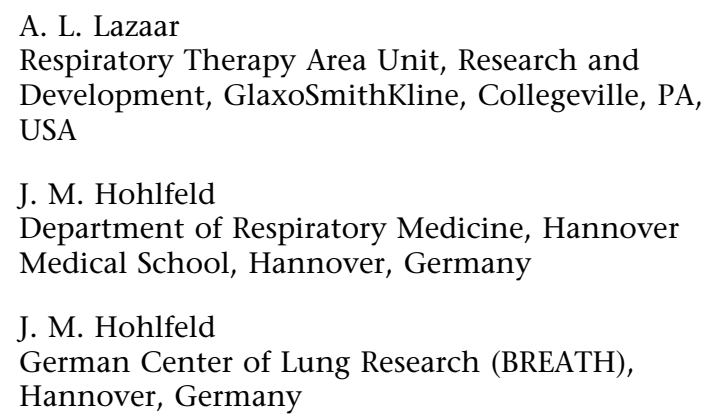


Following GSK2586881 administration, levels of the RAS peptide angiotensin II decreased while angiotensin (1-7) increased, as expected, indicating that GSK2586881 was pharmacologically active.

Conclusions: A single IV dose of GSK2586881 $0.8 \mathrm{mg} / \mathrm{kg}$ was well tolerated but did not impact the acute HPV response in healthy volunteers.

Keywords: Acute hypoxia; Exercise; GSK2586 881; Hypoxic pulmonary vasoconstriction; Pharmacodynamics; Pharmacokinetics; Reninangiotensin system; Recombinant human angiotensin-converting enzyme 2 ; rhACE2

\section{Key Summary Points}

Why carry out this study?

Enhancing activity of the angiotensinconverting enzyme 2 (ACE2), a key enzyme of the renin-angiotensin system (RAS), using the recombinant form of human ACE 2 could be beneficial in diseases such as acute respiratory distress syndrome but may compromise the hypoxic pulmonary vasoconstriction (HPV) and potentially impact systemic and tissue oxygenation.

The exact role of the RAS in modulating the acute HPV response is currently unclear.

This study evaluated the effect of GSK2586881, a recombinant form of human ACE2, on the HPV response and subsequent potential impact on systemic oxygen saturation in healthy adult volunteers during exercise under hypoxic conditions.

\section{What was learned from the study?}

A single IV dose of GSK2586881 $0.8 \mathrm{mg} / \mathrm{kg}$ was well tolerated but did not impact the acute HPV response in healthy volunteers.

This research contributes to scientific dialogue, and publication will further understanding in this field. Although the study was terminated early, the results demonstrated that HPV occurs independently of RAS peptides under the experimental conditions tested.

\section{INTRODUCTION}

The renin-angiotensin system (RAS) may play an important role in controlling pulmonary blood flow via modulation of pulmonary vessel tone [1] and by regulating pulmonary vascular remodelling $[2,3]$. The key effector peptide of the RAS, angiotensin II (Ang II), acting via the angiotensin type $\mathrm{I}$ receptor $\left(\mathrm{AT}_{1} \mathrm{R}\right)$, has been shown to act as a potent pulmonary vasopressor and pulmonary vascular mitogen $[3,4]$.

The membrane-bound carboxypeptidase angiotensin-converting enzyme 2 (ACE2) functions to hydrolyse Ang II to the biologically active heptapeptide angiotensin (1-7) (Ang 1-7). Thus, ACE2 regulates RAS signalling, both directly by reducing Ang II signalling and indirectly by activating the counter-regulatory Ang 1-7 pathway [5]. The Ang 1-7 pathway appears to elicit pulmonary vasoactivity, but in contrast to the vasopressor and mitogenic properties of the Ang II pathway, Ang 1-7 inhibits pulmonary vascular remodelling [6] and promotes vasodilatory responses [7].

For cardiopulmonary diseases where blood flow to the lungs is impaired (such as pulmonary arterial hypertension [PAH] [8] and acute respiratory distress syndrome [ARDS] with associated right heart dysfunction [9]), agents that are capable of alleviating the afterload on the right ventricle, achieved through pulmonary vasorelaxation and/or inhibition of pulmonary vascular remodelling, might be therapeutically beneficial. Thus, boosting the levels and/or activity of ACE2 may represent one such strategy.

In the context of diseases such as ARDS, the use of pulmonary vasodilators to alleviate right ventricular afterload needs to be approached with caution, as they may also compromise hypoxic pulmonary vasoconstriction (HPV) if the impact on pulmonary vascular tone is not restricted to areas that are adequately ventilated [10]. The net effect of non-selective pulmonary vasodilatation on ARDS patients could be catastrophic, as systemic hypoxaemia may be exacerbated due to further mismatching of ventilation and perfusion [10]. 
The exact role of the RAS in modulating the acute HPV response in preclinical species and humans is currently unclear. Preclinical studies have generated conflicting evidence regarding the role of the RAS in mediating acute HPV $[4,11,12]$, whereas the majority of clinical studies have indicated an important role for the RAS in mediating acute HPV in healthy volunteers $[1,13]$. Soluble, recombinant human ACE2 (rhACE2) administration in pigs exposed to acute hypoxia has shown that HPV is regulated by this axis [14]. Interestingly, in addition to Ang II, the plasma levels of other ACE2 peptide substrates have been shown to be regulated by exposure to acute hypoxia in humans [15]. Taken together, these data indicate a potentially important role for ACE2 in modulating HPV in humans.

GSK2586881 is a purified intravenous (IV) formulation of rhACE2. While there is a biological rationale for modulation of the RAS as a means to lessen pulmonary vascular dysfunction in ARDS patients, therapeutic strategies aimed at modulating the RAS may have unintended, negative consequences if they result in ventilation-perfusion mismatching, and consequently, reduction in peripheral oxygen saturation levels. Thus, we conducted a phase I study using an acute hypoxia and exercise challenge model in healthy volunteers to investigate the safety and physiological impact on the acute HPV response by GSK2586881 and subsequent potential impact on systemic oxygen saturation.

The present study reported the safety, pharmacokinetics (PK) and pharmacodynamics (PD) of GSK2586881 in healthy volunteers during an exercise challenge under hypoxic conditions. The primary objective was to evaluate the effect of a single IV dose of GSK2586881 on the HPV response in healthy volunteers during exercise under hypoxic conditions. Secondary objectives were to evaluate (1) the effect of a single IV dose of GSK2586881 on circulating RAS peptide concentrations, (2) the safety of a single IV dose of GSK2586881 in healthy volunteers and (3) the PK of a single IV dose of GSK2586881.

\section{METHODS}

\section{Study Design}

The study was a phase I, single-centre, randomised, double-blind (sponsor open), placebocontrolled, two-period crossover study conducted at the Fraunhofer Institute for Toxicology and Experimental Medicine in Germany (GlaxoSmithKline study 204987; NCT03000686).

Following a screening period of up to 28 days, participants were randomly assigned to one of two treatment sequences comprising two treatment periods, $\mathrm{AB}$ or $\mathrm{BA}$, where $\mathrm{A}$ was placebo and $B$ was GSK2586881 $0.8 \mathrm{mg} / \mathrm{kg}$, administered as a single IV dose infused over 3-5 min. The two treatment periods were separated by a minimum washout period of 3 days to ensure that biomarkers returned to baseline values, and a maximum period of 14 days to give participants flexibility on the dates for their second study period.

The study was double-blinded: participants and non-sponsor study staff were blinded to treatment arms, but limited pre-specified sponsor staff were unblinded. A pre-specified interim analysis was conducted when 10 participants had completed both treatment periods. Following a protocol amendment based on the results of this pre-specified interim analysis, the study was split into two sequential parts: before (Part 1) and after (Part 2) this interim analysis.

In Part 1 of the study, a simulated altitude of $4000 \mathrm{~m} \pm 10 \%$ was achieved in a temperaturecontrolled normobaric hypoxia chamber for the hypoxia and exercise challenge. Participants exercised on an upright cycle ergometer (Ergoline 900, Bitz, Germany) at $70 \%$ of maximum rate of oxygen consumption $\left(\mathrm{VO}_{2 \max }\right)$ within the hypoxia chamber for $10 \mathrm{~min}$ (minimum $5 \mathrm{~min}$ ). Echocardiogram measurements were performed pre-dose (T0), 15 min post-infusion (T1), 60 min after chamber entry (T2), immediately after exercise (T3) and $30 \mathrm{~min}$ after chamber exit (T4) (Fig. 1).

In Part 2, a simulated altitude of $5000 \mathrm{~m} \pm 10 \%$ was achieved in the chamber. The participants exercised on a semi-recumbent 
cycle ergometer (Ergoselect 1200 ELP) tilted by $30-40$ degrees at $50 \%$ of $V_{2}$ max within the hypoxia chamber. Participants exercised for a minimum of $5 \mathrm{~min}$ and until the echocardiogram recording was complete. Measurements were performed at the time points as in Part 1, except for the T3 echocardiogram, which was performed $2 \mathrm{~min}$ into the exercise challenge (rather than at the end of the 10-min exercise challenge). Due to significant variation in the HPV response reported in healthy subjects [16], modifications in chamber altitude and exercise protocol were made to enhance participant hypoxia and thus increase the pulmonary artery systolic pressure (PASP) measured during the hypoxia and exercise challenge. Participants remained in the hypoxia chamber for approximately 80 minutes per period.
All participants provided written informed consent, and the independent ethics committee of the Hannover Medical School reviewed and approved the study. The study was conducted in accordance with the Declaration of Helsinki, the International Council on Harmonisation, Good Clinical Practice and local regulations.

\section{Participants}

The study population comprised adult volunteers 18-40 years of age, with a screening echocardiogram showing mild-moderate tricuspid regurgitation sufficient for reliable estimation of PASP. Participants had a body weight of $50-100 \mathrm{~kg}$ (inclusive), had not resided at an altitude of $>1500 \mathrm{~m}$ for $>7$ days in the last 4 months and had no contraindication to performing exercise on a bicycle ergometer. Full

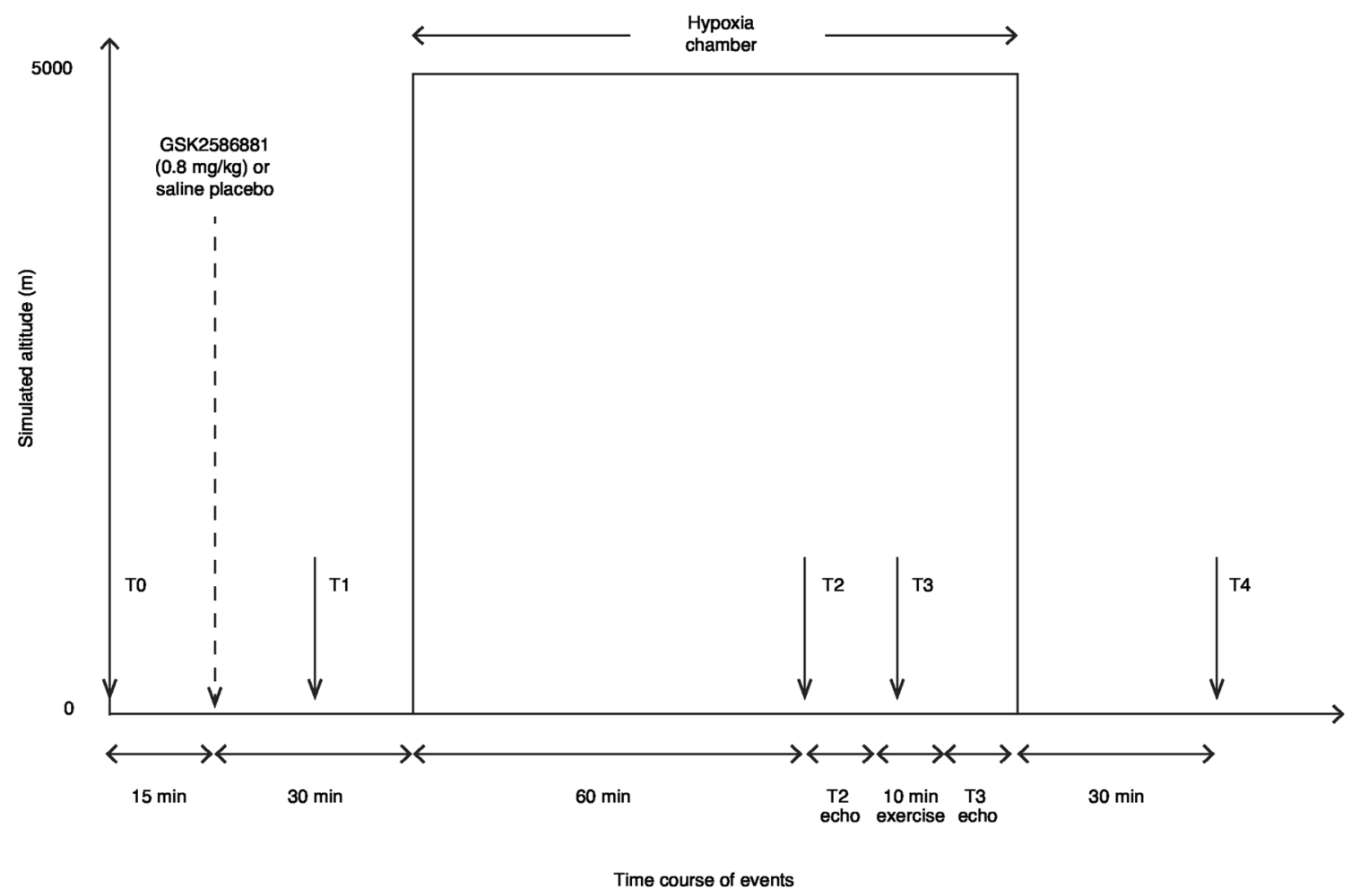

Fig. 1 Study design and key study features. Timing of simulated altitude and echocardiogram measurements (black arrows) during treatment periods 1 and 2. The schematic represents the optimised design in Part 2 of the study but is also applicable to Part 1 with the following modifications: simulated altitude of $4000 \mathrm{~m}$ and T3 echocardiogram taken at the end of the 10-min exercise challenge. T0, pre-dose; T1, 15 min post-infusion; T2, 60 min after chamber entry; T3, 2 min after exercise start (Part 2); T4, 30 min after chamber exit. $T$ treatment time point 
inclusion and exclusion criteria are available at ClinicalTrials.gov (NCT03000686).

Between 17th May 2017 and 4th January 2019, 48 participants were screened, and 31 $(65 \%)$ failed screening, primarily for not meeting the eligibility criteria of mild-moderate tricuspid regurgitation. Therefore, 17 participants were randomised to treatment. In each treatment period, GSK2586881 was administered as a slow infusion over $3-5 \mathrm{~min}$. All participants received $0.8 \mathrm{mg} / \mathrm{kg}$ GSK2586881, except for one participant who received $0.6 \mathrm{mg} / \mathrm{kg}$ in error; this participant was included in the safety and efficacy analyses.

\section{Endpoints and Assessments}

The primary endpoint of the study was change in PASP from baseline, as assessed by echocardiography. Secondary endpoints were: (1) the effect of GSK2586881 on RAS peptides (e.g. Ang II, Ang 1-7, Ang 1-5) at baseline and in response to hypoxia and exercise, (2) the effect of GSK2586881 on vital signs (heart rate, systolic and diastolic blood pressure), 12-lead electrocardiograms (ECGs), clinical laboratory assessments and continuous pulse oximetry, adverse events (AEs) and immunogenicity, and (3) plasma concentrations of GSK2586881 and derived PK parameters.

Echocardiograms were performed at four time points (T1-T4) and the modified Bernoulli equation was used to determine PASP across the treatment period (Fig. 1; Table S1). Oxygen saturation (by pulse oximetry) and ECG monitoring (by telemetry) were performed continuously throughout the treatment period. Safety assessments included vital signs, 12-lead ECGs, clinical laboratory tests, physical examinations and the number and type of AEs, all of which were performed at various time points from study screening until final follow-up 7-10 days after the last dose. PK assessment of GSK2586881 by immunoassay, and PD/biomarker assessments of RAS peptides (Ang II, Ang 1-7 and Ang 1-5) and renin by a method based on reversed-phase solid-phase extraction, were performed as previously described [17] at eight time points across the treatment period (from pre-dose to
30 min after chamber exit) (Table S1). Critical reagents in the assay for determining GSK2586881 concentration in plasma (reference material, coating material and detection reagents) were changed between Parts 1 and 2 of the study. Immunogenicity was assessed by immunoassay for anti-ACE2 binding antibodies at T0 and at the final follow-up.

The modified intent-to-treat (mITT) population, defined as all randomised participants, excluding those who were randomised in error, was the primary population for all safety and pharmacodynamic analyses. The mITT1 and mITT2 populations comprised all participants randomised to Part 1 (4000 m altitude) or Part 2 (5000 $\mathrm{m}$ altitude) of the study, respectively.

\section{Statistical Analysis}

Based on study feasibility, the starting sample size was 25 enrolled participants for 20 evaluable. Simulation was used to estimate the precision of the primary endpoint (change in PASP from baseline), with consideration given to a clinically relevant treatment-related reduction of $5 \mathrm{mmHg}$.

Following the Part 1 pre-specified interim analysis (10 participants completing the two treatment periods), the decision was made for the study to continue, but with modifications to the protocol to optimise the hypoxia/exercise model and improve the quality of the echocardiogram data (Part 2). In Part 2, two further interim analyses were performed after three participants and after six participants had completed both treatment periods. The aim of these interim analyses was to assess the effectiveness of the protocol modifications on critical assessments and potential benefit-risk impact on participants. Predefined stopping criteria, based on PASP variability and deviation from the expected placebo profile (increase during hypoxia and during exercise) or safety data, allowed the study to be stopped at either interim analysis in Part 2.

No formal statistical hypotheses were tested in either Part 1 or Part 2. A Bayesian repeatedmeasures mixed-effects regression model with non-informative priors for model parameters 
was used to model the change from baseline in PASP, with T3 being the primary time point of interest. Posterior distributions were used to produce posterior probability statements about the magnitude of treatment effects; for example, the probability that the treatment difference for change from baseline in PASP (GSK2586881-placebo) was $\leq 5 \mathrm{mmHg}$ (>5 mmHg in magnitude). Separate analyses were performed for Part 1 and Part 2 (details are provided in the electronic supplementary material). Similar methods of analysis were applied to relevant secondary endpoints. Biomarker data were log-transformed prior to analysis.

The software used for statistical analysis was SAS v9.4 (TS1M3), running on Linux. SAS PROC MCMC was used to conduct the Markov chain analyses. Plasma GSK2586881 concentration-time data were analysed by non-compartmental methods using Phoenix WinNonlin 8.1.

\section{RESULTS}

\section{Study Population}

A total of 48 participants were screened. Of these, 17 (35\%) participants meeting the eligibility criteria were randomised to treatment (Fig. 2). As described, a pre-specified interim analysis was conducted when 11 participants had been randomised, and 10 had completed both treatment periods (Part 1). These results demonstrated that the model did not produce sufficient changes from pre-dose to hypoxic/ exercised PASP, requiring alterations to the study design. Following a protocol amendment to optimise the experimental induction of hypoxia, six additional participants were randomised for Part 2, and all completed the study. In total, 16/17 participants completed the study as planned (Fig. 2). Table 1 summarises the demographics and baseline characteristics for the mITT1 and mITT2 populations, which were similar between these populations.

The study was terminated following review of PASP data at the second interim analysis of Part 2, which showed that futility was met according to pre-specified success criteria.
Termination was also based on the technical feasibility of the study, including the challenges of obtaining echocardiogram recordings during exercise.

\section{Efficacy and Safety}

\section{Pulmonary Artery Systolic Pressure}

In Part 1, changes in PASP were inconsistent versus expectations at $\mathrm{T} 2$ and $\mathrm{T} 3$ in participants receiving placebo treatment. Individual participant profiles for change from baseline in PASP were variable, with the increases at T3 (hypoxia plus exercise) being lower than those at T2 (hypoxia alone) (data not shown). The inability to reliably detect an HPV response suggested that the hypoxia model was not performing as expected. Therefore, no conclusions could be drawn for the primary endpoint, and the protocol was amended to optimise the hypoxia/ exercise model and improve the quality of the echocardiographic data.

In Part 2, the chamber was modified to an altitude of $5000 \mathrm{~m}$ to increase participant hypoxia, and T3 PASP was measured at 2 min into the exercise challenge, rather than after completion of a 10-min challenge. This change was based on evidence demonstrating that prolonged steady-state exercise (even $10 \mathrm{~min}$ ) can conversely decrease pulmonary vascular pressures due to a reduction in pulmonary blood volume as the systemic circulation adapts [18].

The results for the placebo arm showed that the protocol changes had significantly improved the HPV model as shown by the increase in PASP at both T2 and T3 (Fig. 3); however, no clinically relevant differences between the treatment arms were observed. The statistical model showed that the change from baseline in PASP was $0.018 \mathrm{mmHg}$ and $2.796 \mathrm{mmHg}$ lower for GSK2586881 compared with placebo at $\mathrm{T} 3$ and $\mathrm{T} 2$, respectively (Table 2). The potential difference observed at T2 appeared to be driven primarily by the results of two individual participants (data not shown). The posterior probability of observing a reduction $>5 \mathrm{mmHg}$ at $\mathrm{T} 3$ was $11 \%$ and at $\mathrm{T} 2$ was $24 \%$ (Table 2 ). 


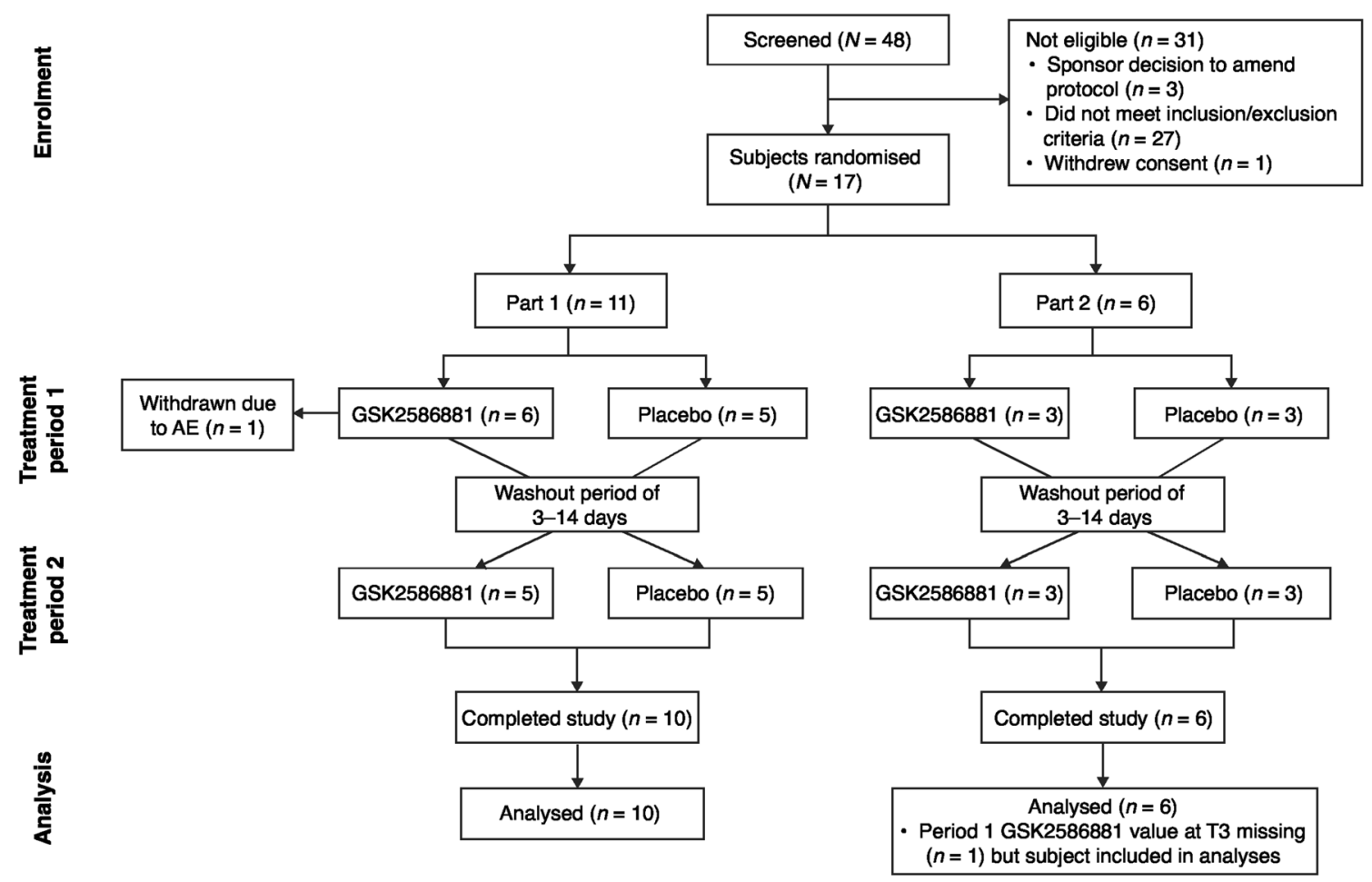

Fig. 2 Treatment flow. Note: The mITT2 (Part 2) population $(n=6)$ were randomised after interim analysis of the mITT1 (Part 1$)$ population $(n=11)$. mITT modified intent-to-treat

\section{Oxygen Saturation}

In Part 1, the oxygen saturation profiles for GSK2586881 and placebo were similar, with decreases observed from T1 to T3, confirming that the hypoxia chamber was operating as expected (Fig. 4). In Part 2, further decreases in oxygen saturation were observed for both treatment arms (Fig. 4). Overall, there were no clinically relevant treatment differences in oxygen saturation measured by pulse oximetry between GSK2586881 and placebo in either part of the study.

Vital Signs, 12-Lead ECGs, Immunogenicity, Clinical Laboratory Assessments and AEs

In both study parts, mean vital signs (systolic and diastolic blood pressure, heart rate and temperature) were similar between treatment groups, and changes from baseline were not clinically relevant; increases in heart rate following the exercise challenge were as expected. There were no clinically relevant changes in
ECG or laboratory evaluations attributed to study medications, and no evidence of immunogenicity (data not shown).

In Part 1 , seven AEs were reported by $5 / 11$ participants, and in Part 2, one AE was reported by $1 / 6$ participants. In both parts of the study, the incidence of AEs was lower with GSK2586881 (Part 1: 18\%; Part 2: 0\%) than placebo (Part 1: 30\%; Part 2: 17\%) (Table S2). In both parts of the study, all AEs were mild or moderate in intensity, except for one severe $\mathrm{AE}$ (SAE) in Part 1; a participant receiving GSK2586881 in treatment period 1 had a vasovagal reaction (presyncope) that was not considered drug-related but resulted in withdrawal from the study. No other SAEs, deaths or other AEs were reported. 
Table 1 Participant demographics in mITT1 (Part 1) and mITT2 (Part 2) populations

\begin{tabular}{|c|c|c|}
\hline Demographics & $\begin{array}{l}\text { mITT1 } \\
\text { population } \\
(n=11)\end{array}$ & $\begin{array}{l}\text { mITT2 } \\
\text { population } \\
(n=6)\end{array}$ \\
\hline Male, $n$ & 11 & 6 \\
\hline $\begin{array}{l}\text { Age in years, }{ }^{a} \text { mean } \\
\text { (SD) }\end{array}$ & $26.2(4.2)$ & $29.2(6.2)$ \\
\hline Aged $18-40$ years, ${ }^{a} n$ & 11 & 6 \\
\hline $\begin{array}{l}\text { BMI }\left(\mathrm{kg} / \mathrm{m}^{2}\right) \text {, mean } \\
\quad(\mathrm{SD})\end{array}$ & $24.1(1.9)$ & $24.9(2.5)$ \\
\hline $\begin{array}{l}\text { Height }(\mathrm{cm}) \text {, mean } \\
\quad(\mathrm{SD})\end{array}$ & $183.7(6.6)$ & $182.3(3.4)$ \\
\hline $\begin{array}{l}\text { Weight }(\mathrm{kg}) \text {, mean } \\
(\mathrm{SD})\end{array}$ & $81.2(6.8)$ & $82.7(8.8)$ \\
\hline $\begin{array}{l}\text { White/Caucasian/ } \\
\text { European heritage, } \\
n\end{array}$ & 11 & 6 \\
\hline
\end{tabular}

\section{Pharmacodynamics, Biomarkers and Pharmacokinetics}

\section{RAS Peptides and Renin}

In Part 1, Ang II was present at quantifiable concentrations in pre-dose samples from all participants prior to placebo and all but two participants prior to GSK2586881 treatment. Following infusion with GSK2586881, the circulating Ang II concentration fell to undetectable levels in the majority of participants and remained largely so throughout the study period. Following placebo infusion, there was also an unexpected decrease in the circulating Ang II concentration. This decrease was, however, smaller and more variable than that seen after treatment with GSK2586681 and was only maintained until T2. At T3, the concentration of Ang II was elevated relative to pre-dose in all participants in the placebo arm. Following exit from the chamber, the concentration of Ang II decreased towards the pre-dose level. Thus, in
Part 1, there was no increase in circulating Ang II in response to hypoxia alone (T2) but only in response to exercise and hypoxia (T3), as illustrated in Fig. 5. The ratio of the treatment-induced reduction in Ang II compared with placebo was $<1$ at all time points, demonstrating a significant decrease in Ang II levels in the GSK2586881 arm compared with the placebo arm. At T3, there was a greater separation between the treatment arms; the probability of observing a 50\% greater reduction in Ang II compared with placebo was $97 \%$.

In Part 1, it was not possible to model the Ang 1-7 results statistically due to the high number of values below the lower limit of quantification (LLOQ). However, Ang 1-7 geometric mean concentrations remained at low or undetectable levels after placebo infusion, while they increased continually following GSK2586881 infusion until after chamber exit, then returned towards baseline levels at T4 (Fig. 5). Ang 1-5 levels showed the expected similar pattern of change to Ang 1-7. They were below LLOQ in all participants before treatment and remained at low or undetectable levels following placebo throughout the measurement period. Ang 1-5 concentrations increased from T1 to T3 with GSK2586881, peaking postchamber exit and returning towards baseline levels at T4. The ratio of the change in Ang 1-5 with GSK2586881 treatment compared with placebo was $>1$ for all time points. At T3, the probability of an increase in Ang 1-5 greater than $75 \%$ compared with placebo was $100 \%$.

As expected, changes in the concentration of renin, the key rate-determining enzyme in the RAS, broadly reflected the changes in Ang II concentration after placebo treatment and in Ang 1-7 and Ang 1-5 concentration after GSK2586881 treatment (Fig. 5). The time of peak renin levels in each arm corresponds to the time of the peak levels of the relevant RAS peptide. Therefore, treatment with GSK2586881 delayed the peak in renin production relative to placebo. Additionally, the concentration of renin at T2, T3 and post-chamber exit was greater after GSK2586881 than placebo.

In Part 2, following treatment with GSK2586881 and placebo, Ang II, Ang 1-7, Ang 1-5 and renin showed changes in concentration 


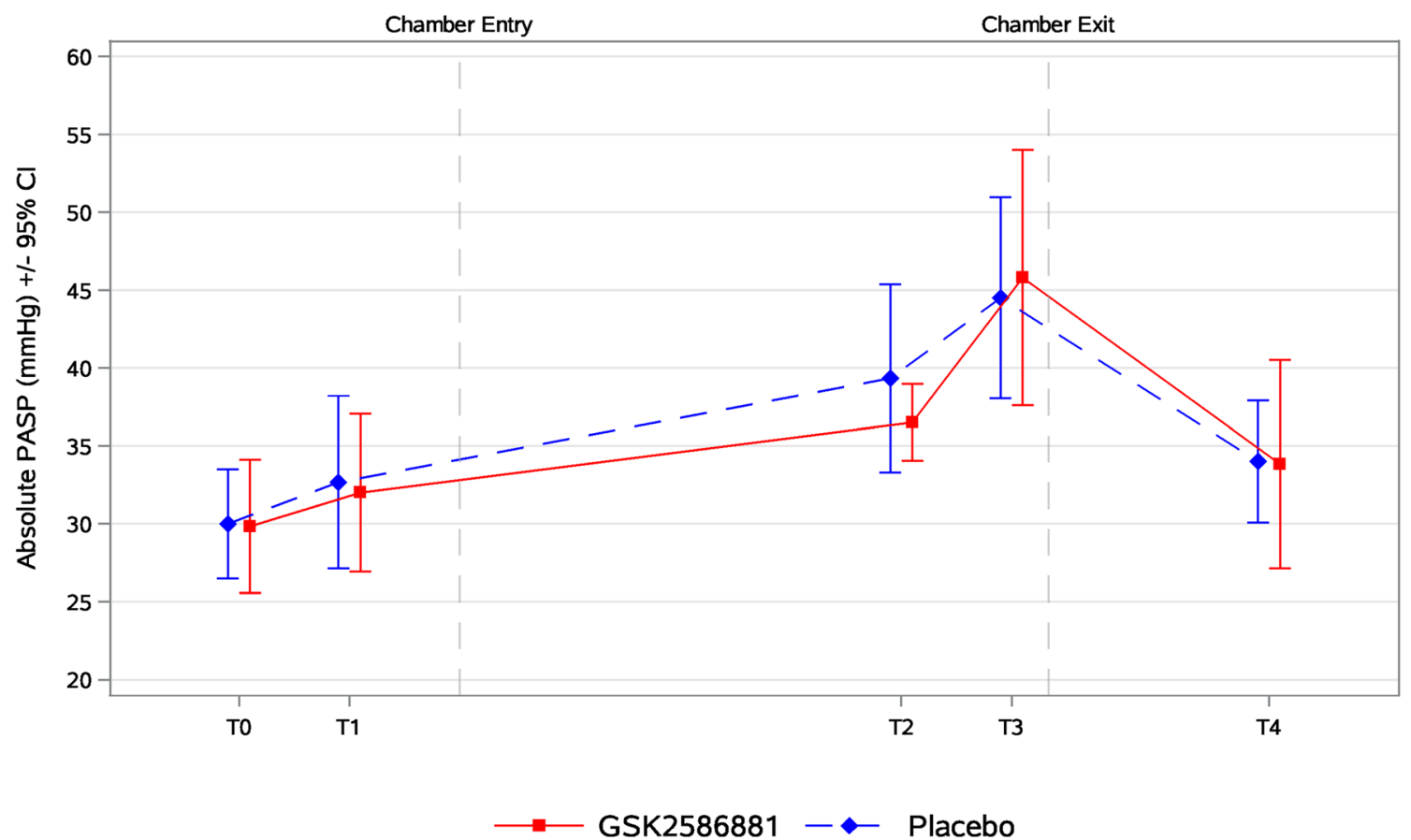

Fig. 3 Absolute PASP following treatment with GSK2586881 or placebo. Data are for the mITT2 population (Part 2). T0, pre-dose; T1, $15 \mathrm{~min}$ postinfusion; T2, 60 min after chamber entry; T3, 2 min after exercise start; T4, 30 min after chamber exit. $C I$ confidence interval, $m I T T$ modified intent-to-treat, $P A S P$ pulmonary artery systolic pressure, $T$ treatment time point

Table 2 Repeated-measures Bayesian statistical analysis of change from baseline in PASP $(\mathrm{mmHg})$

\begin{tabular}{|c|c|c|c|c|c|c|}
\hline \multirow[t]{2}{*}{ Time point } & \multirow[t]{2}{*}{ Posterior median difference (SD) } & \multirow[t]{2}{*}{$95 \%$ credible interval } & \multicolumn{4}{|c|}{ Posterior probability of true difference (\%) } \\
\hline & & & $<0$ & $<-2.5$ & $<-3$ & $<-5$ \\
\hline $\mathrm{T} 1$ & $-0.662(2.193)$ & $(-5.037,3.815)$ & 63.60 & 17.38 & 12.13 & 2.57 \\
\hline $\mathrm{T} 2$ & $-2.796(3.430)$ & $(-9.729,4.027)$ & 81.26 & 53.83 & 47.39 & 24.11 \\
\hline T3 & $-0.018(4.145)$ & $(-8.475,8.086)$ & 50.18 & 26.13 & 22.24 & 10.75 \\
\hline $\mathrm{T} 4$ & $-0.291(2.328)$ & $(-4.960,4.386)$ & 55.59 & 15.28 & 10.82 & 2.42 \\
\hline
\end{tabular}

mITT modified intent-to-treat, $P A S P$ pulmonary artery systolic pressure, $S D$ standard deviation

Treatment comparisons between GSK2586881 and placebo in the mITT2 (Part 2) population are reported

largely consistent with those observed in Part 1. Thus, Ang II decreased to undetectable levels after GSK2586881 and showed a more modest, variable decrease after placebo treatment, while Ang 1-7 and Ang 1-5 increased after infusion of GSK2586881 but remained at undetectable levels after placebo. In contrast to Part 1, however, Ang II did not increase after hypoxia plus exercise, and the concentrations of Ang 1-7 and Ang 1-5 after GSK2586881 remained stable after $\mathrm{T} 1$ rather than increasing during the period in the hypoxic chamber. Thus, in Part 2, there was no impact of hypoxia alone or combined with exercise on RAS peptide concentrations. Renin concentration also showed little change in response to hypoxia or hypoxia plus exercise, although it was increased 


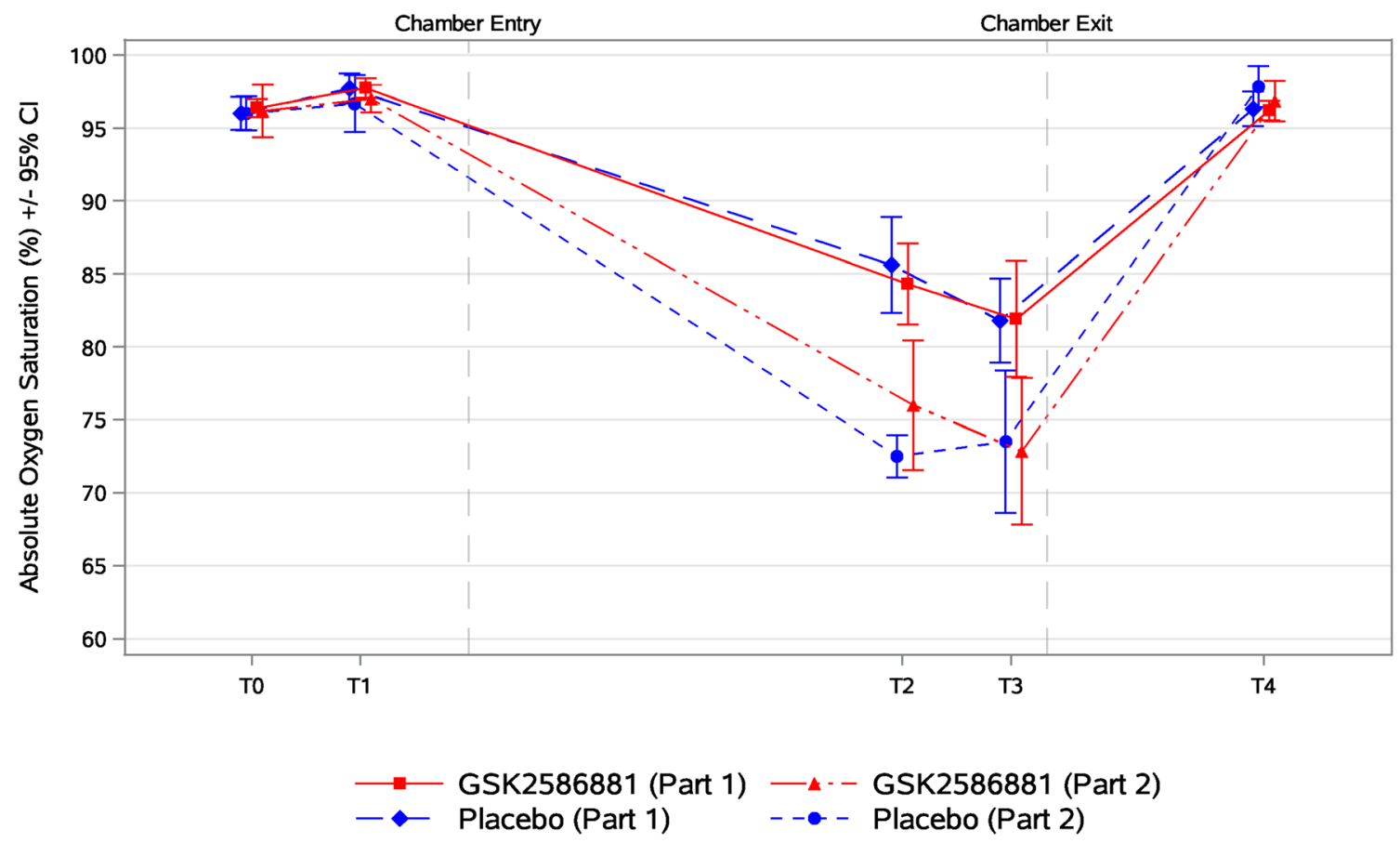

Fig. 4 Oxygen saturation following treatment with GSK2586881 or placebo. Data reported for the mITT1 (Part 1) population and mITT2 (Part 2) population. T0, pre-dose; T1, $15 \mathrm{~min}$ post-infusion; T2, $60 \mathrm{~min}$ after

after GSK2586881 treatment compared with placebo.

\section{Plasma Concentrations and Pharmacokinetics of GSK2586881}

For all participants who completed the hypoxia challenge, GSK2586881 was quantified ( $>200 \mathrm{ng} / \mathrm{mL}$ ) in plasma up to $30 \mathrm{~min}$ after hypoxia chamber exit in both study parts. In 10/17 participants, maximum concentrations $\left(C_{\max }\right)$ were observed in samples collected as soon as possible after the IV infusion of GSK2586881 (3-6 min) (Fig. 6). However, in some subjects (Part 1: 6/11; Part 2: 1/6) $C_{\max }$ was delayed to $15-30 \mathrm{~min}$ post-infusion. These observations are inconsistent with the infusion times which were recorded as $\leq 6 \mathrm{~min}$ in all participants.

$C_{\max }$ and systemic exposure over the entire study (as measured by geometric mean area under the curve [AUC] [0-2.5 h]), and during the hypoxia challenge (AUC [0.5-2 h]), was 1.6to 1.8-fold higher in Part 2 compared with Part chamber entry; T3, immediately after exercise (Part 1)/ 2 min after exercise start (Part 2); T4, $30 \mathrm{~min}$ after chamber exit. $C I$ confidence interval, $m I T T$ modified intent-to-treat, $T$ treatment time point

1 , which may in part be due to the assay reagent changes. In both study parts, the between-subject variability was low for both AUC and $C_{\max }$.

\section{DISCUSSION}

The purpose of this study was to investigate whether a single IV dose of recombinant human ACE2 GSK2586881 $(0.8 \mathrm{mg} / \mathrm{kg})$ could modulate the acute HPV response in healthy volunteers, and if there was a subsequent impact on oxygen saturation. A hypoxia/exercise challenge model was used to reduce alveolar oxygen tension with a consequential increase in pulmonary arterial pressure. The anticipated result in the placebo arm was an increase in PASP at T2 (60 min after hypoxia chamber entry) and a further increase at T3 (hypoxia plus exercise). GSK2586881 was well tolerated in this study, with a safety profile consistent with placebo; however, the study did not meet its primary endpoint of change from baseline in PASP. In Part $1(4000 \mathrm{~m} ; 10$ 


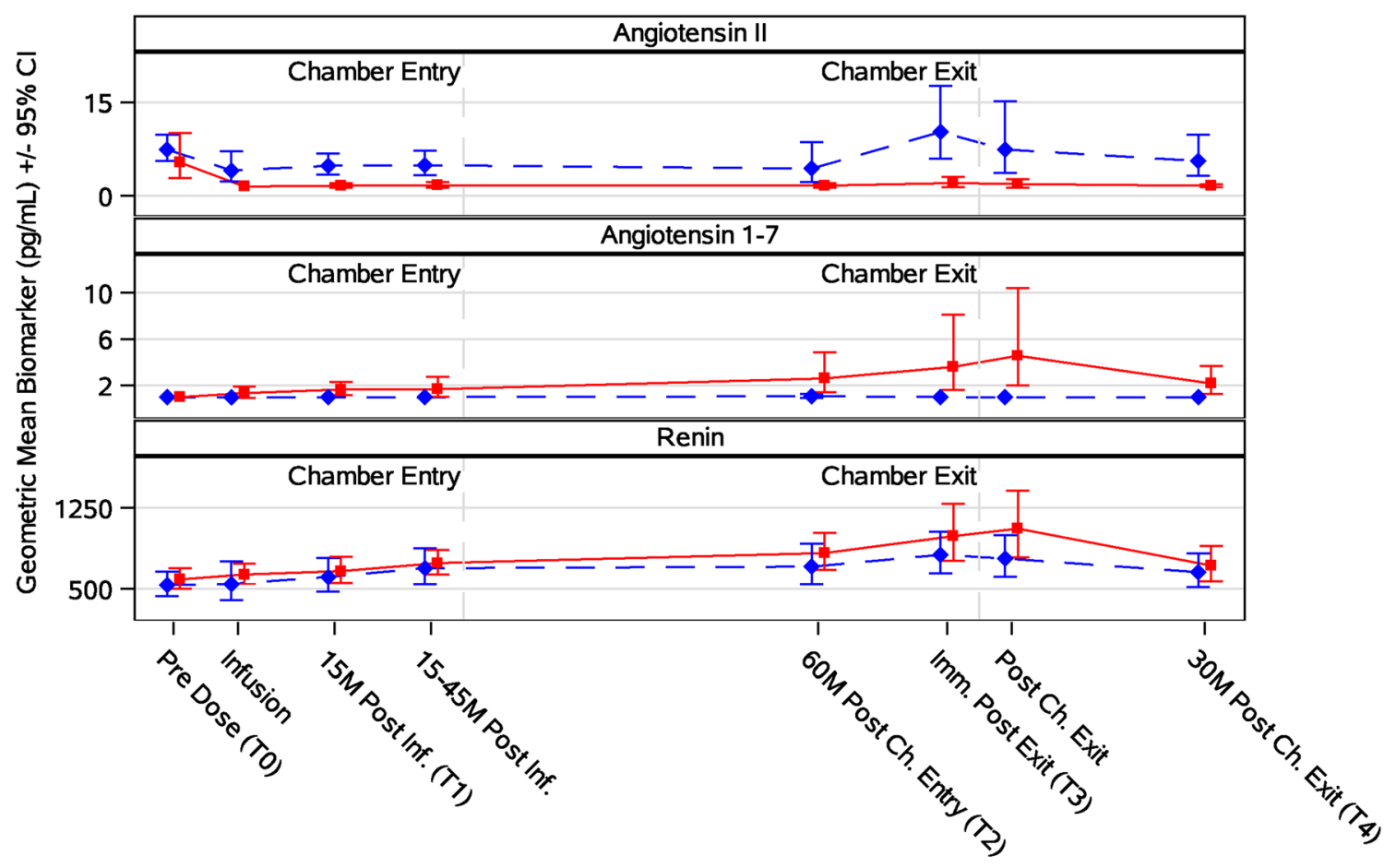

$\longrightarrow$ GSK2586881 —ઐ Placebo

Fig. 5 Time profiles from baseline in RAS peptides following treatment with GSK2586881 or placebo. Time profiles of Ang II, Ang 1-7 and renin reported in the mITT1 (Part 1) population. Ang angiotensin, Ch.

completed participants), there was no consistent increase in PASP at T2 and T3 with placebo, and individual participant profiles were variable, likely reflecting different sensitivities to reduced alveolar oxygen levels. Therefore, no reliable conclusions could be made about any potential treatment effect.

The results of the placebo arm in Part 2 showed that modifications to the hypoxia challenge and exercise protocol made detection of HPV more robust. The magnitude of increase in PASP and the decrease in oxygen saturation was similar to changes reported by Ricart et al. [19] using similar hypoxia conditions as in Part 2. Despite this, there were no clinically relevant treatment differences in PASP from baseline between treatment arms following exercise. From a safety perspective, no acute worsening of hypoxaemia was observed in participants chamber, CI confidence interval, Ent. entry, Inf. infusion, $I m m$. immediately, $M$ minutes, $m I T T$ modified intent-totreat, $T$ treatment time point

who received GSK2586881 prior to the exercise challenge.

The efficacy data on pulmonary artery pressures contrast with those from Hemnes et al., where treatment with GSK2586881 resulted in a modest reduction in pulmonary vascular resistance in patients with PAH [20]. This may be due to differences in Ang II levels seen in PAH compared with healthy subjects undergoing hypoxia challenge or in the sensitivity of the methods used to determine PASP (catheterisation vs echocardiogram). Technical issues may also have contributed to the lack of observed effect. Although a semi-recumbent ergometer was used to allow recording of echocardiograms during (rather than after) exercise, in practice, the participant was required to stop exercising in order to obtain an echocardiogram of sufficient quality to measure PASP. In healthy 


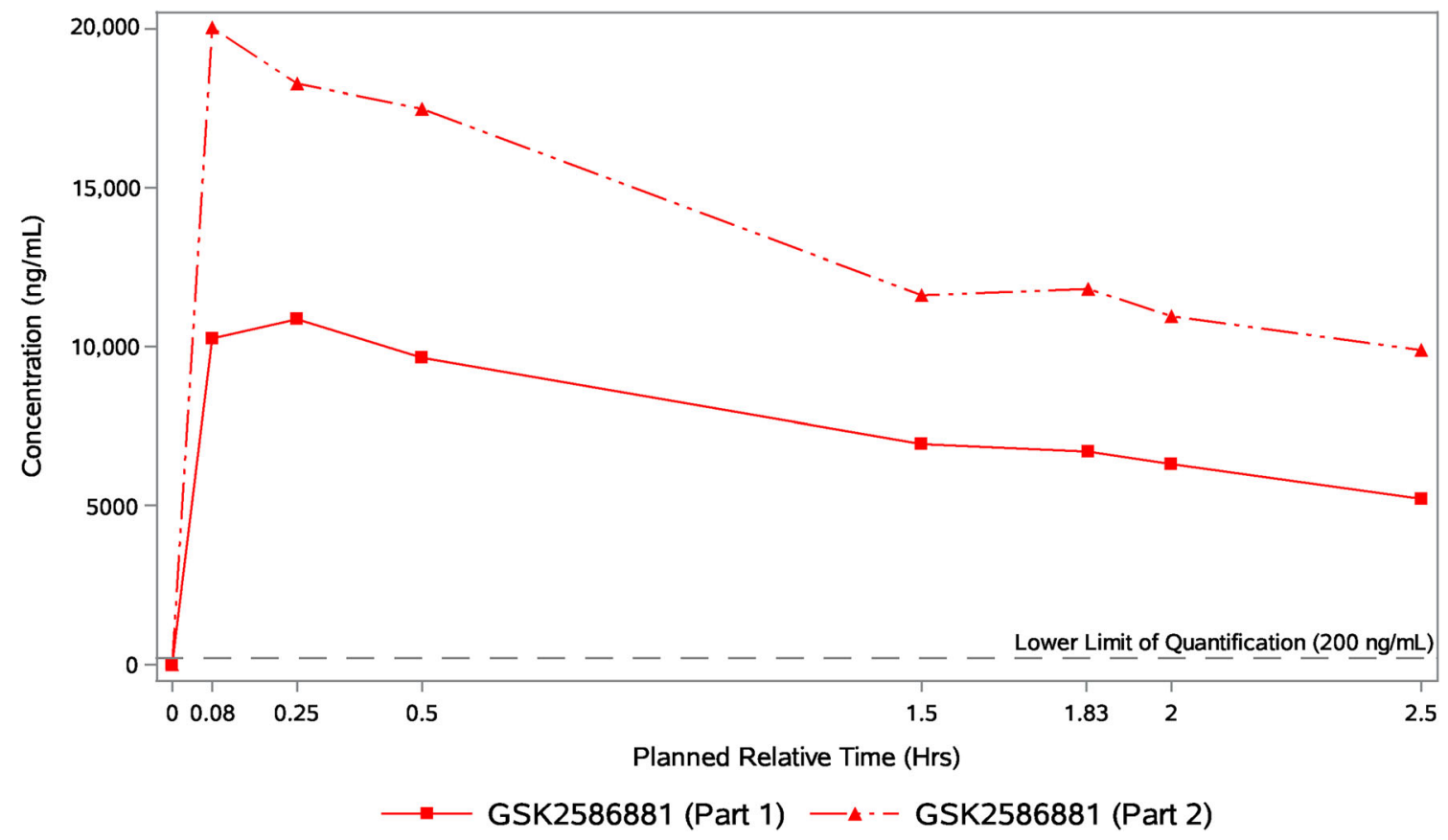

Fig. 6 Mean GSK2586881 plasma concentrations. Data reported for the mITT1 population (Part 1) and mITT2 (Part 2) populations. Note: Planned relative time of $0 \mathrm{~h}$

volunteers, this cessation of exercise may have resulted in a rapid return to baseline, thus obscuring any potential drug effect. The observed pharmacokinetics of GSK2586881 along with the rapid modulation of RAS peptides (discussed below) suggest that the lack of effect on pulmonary artery pressures cannot be attributed to inadequate exposure.

Target engagement of GSK2586881 was evaluated by measurement of key RAS peptides. ACE2 is known to cleave Ang II to Ang 1-7, with further cleavage to Ang 1-5. There was a clear reduction in Ang II levels in participants receiving GSK2586881 compared with placebo, with a corresponding increase in Ang 1-7 and Ang 1-5 concentrations. Interestingly, Ang 1-7 and Ang 1-5 concentrations after GSK2586881 treatment peaked at a later time point (following exit from the hypoxia chamber) than after placebo treatment (peak at T3). A similar change in the time of the peak concentration was seen for renin after GSK2586881 treatment, suggesting it may be a consequence of the loss of feedback inhibition of renin release by Ang II after GSK2586881. Hypoxia plus exercise did represents pre-dose sampling and planned relative time of $0.08 \mathrm{~h}$ represents sample taken at the end of the infusion. mITT modified intent-to-treat

not increase Ang II in Part 2 in contrast to Part 1, and the GSK2586881-induced increase in Ang 1-7 and Ang 1-5 was not further elevated at T2 and $\mathrm{T} 3$. The latter could be a consequence of the reduction of the length and workload of the exercise challenge in Part 2. The rapid modulation of RAS peptides by GSK2586881 provides clear evidence of target engagement and is consistent with previous data obtained in healthy volunteers and ARDS patients $[17,21]$.

It is noteworthy that acute exposure to hypoxia did not result in an increase in the circulating Ang II concentration in either part of this study. Despite this, there was a clear increase in PASP in response to the more severe hypoxia in Part 2. Furthermore, GSK2586881 reduced the circulating concentration of Ang II to below LLOQ $(3 \mathrm{pg} / \mathrm{mL})$ in most participants in both parts of the study, but had no significant impact on PASP compared with placebo. Both observations argue against a major role for circulating Ang II as the mediator of HPV under our experimental conditions. However, we cannot exclude the possibility of a local increase in Ang II concentration in the lung which was 
not reflected in the systemic circulation, although this should also have been susceptible to degradation by GSK2586881, unless it was in an inaccessible compartment. The lack of impact of GSK2586881 treatment on PASP in the present study contrasts with the previously reported effects of $\mathrm{ACE}$ inhibition or $\mathrm{AT}_{1} \mathrm{R}$ blockade on HPV [1, 22], in which reductions of approximately $6 \mathrm{mmHg}$ in mean pulmonary arterial pressure measured by echocardiography were observed in response to acute hypoxia. The difference is likely to be due to the differences in the study protocols and subsequent differences in the effects on the RAS and/or the physiology of the lung. The Lipworth group treated participants with the small molecule RAS modulators for 4 days before exposure to hypoxia via inhalation of a hypoxic mixture of $\mathrm{N}_{2}$ and $\mathrm{O}_{2}$. Their drug treatment protocol resulted in a marked increase in normoxic plasma renin activity but only a limited change in Ang II concentrations [1]. No change in plasma renin activity or Ang II was seen in response to hypoxia after placebo or active treatment, consistent with our data. In the present study, a single dose of GSK2586881 was used, which had a marked impact on Ang II concentrations but only a modest impact on renin concentration under normoxic conditions; however, renin concentration showed some response to hypoxia and exercise.

Most recently, rhACE2 has been tested as a therapeutic for SARS-CoV2 infection, where it may inhibit the binding of the virus spike protein. RhACE2 could be therapeutically useful in other diseases, including many cardiovascular conditions, where the pathophysiological mechanisms driving disease are mediated via the non-classical RAS, providing an opportunity for further future clinical development. The data from the current study indicate that HPV is not mediated via the non-classical RAS under the experimental conditions tested (hypoxia and exercise) in healthy volunteers, negating our ability to assess the impact of attenuating HPV on systemic oxygen saturation with rhACE2. Identification of the pathways responsible for driving HPV in the system used would allow investigation of the clinical question utilising drugs modifying those pathways in future clinical studies, such as PDE5 inhibitors.

A key limitation of the study was that PASP was estimated by echocardiography, rather than a direct measurement of PASP using invasive measures. In echocardiography, PASP is determined by a measured value and an estimated value, making the measurement less robust than direct invasive measurement. A further limitation of the study was the difficulty in obtaining a reliable regurgitation signal via echocardiography to calculate a precise surrogate measure for PASP, resulting in increased variability. Finding healthy volunteers with sufficient tricuspid regurgitation to measure PASP was challenging; several hundred volunteers were pre-screened, and of these volunteers, only 48 were eligible for screening. The study was therefore further limited by the low number of trial participants, significantly increasing the possibility of systematic bias, particularly in Part 2 of the study where only six participants were analysed. The study effects were limited to healthy volunteers, who may have different responses to GSK2586881 than patients with ARDS or PAH, making generalisability of the study findings to these patients challenging.

\section{CONCLUSIONS}

A single IV dose of $0.8 \mathrm{mg} / \mathrm{kg}$ GSK2586881 (rhACE2) was well tolerated in healthy participants, with no SAEs and no evidence of immunogenicity. GSK2586881 did not impact pulmonary vasoconstriction induced by hypoxia, or by hypoxia augmented by exercise, when assessed by echocardiography, and had no consequent effect on peripheral oxygen saturation in healthy participants. GSK2586881 was quantifiable in plasma for the duration of the study $(0-2.5 \mathrm{~h}$ post-dose) in all participants. GSK2586881 caused a robust and sustained reduction in Ang II and a corresponding increase in Ang 1-7 and Ang 1-5, confirming target engagement and pharmacological activity of GSK2586881 in vivo. Hypoxic challenge in healthy participants does not appear to result in robust, detectable alterations in plasma Ang II levels, suggesting that HPV occurs 
independently of Ang II under the experimental conditions tested.

\section{ACKNOWLEDGEMENTS}

The authors would like to thank the participants of the study as well as all study staff for their contributions to the study. The authors are also grateful to Andrew Bayliffe, PhD (GlaxoSmithKline plc at the time of the study, currently Marengo Therapeutics and Apple Tree Partners), who was instrumental in the conception of the study and development of the study design.

Trademarks are owned by or licensed to Certara LP (Phoenix WinNonlin).

Funding. This work was funded by GlaxoSmithKline plc (study 204987; ClinicalTrials.gov identifier: NCT03000686). The journal Rapid Service Fees were funded by GlaxoSmithKline plc.

Medical Writing and/or Editorial Assistance. Medical writing support for the development of this manuscript, under the direction of the authors, was provided by Kirsty Millar, MSc, and Andrew Briggs, BA, of Ashfield MedComms (Macclesfield, UK), an Ashfield Health company, and was funded by GlaxoSmithKline plc.

Authorship. All named authors meet the International Committee of Medical Journal Editors (ICMJE) criteria for authorship for this article, take responsibility for the integrity of the work as a whole, and have given their approval for this version to be published.

Author Contributions. David A. Hall, Kate Hanrott, Philipp Badorrek, David C. Budd, Rhena Eames, William M. Powley, Deborah Hewens, Sarah Siederer, Aili L. Lazaar, Anthony Cahn and Jens M. Hohlfeld contributed to the study conception and design. Philip Badorrek, Dominik Berliner and Jens M. Hohlfeld were involved in data acquisition. David A. Hall, Kate Hanrott, Philipp Badorrek, Dominik Berliner,
Rhena Eames, William M. Powley, Deborah Hewens, Sarah Siederer, Aili L. Lazaar, Anthony Cahn and Jens M. Hohlfeld were involved in data analysis and/or interpretation. All authors were involved in writing/critical review of draft versions of this manuscript and all approved the final version for submission for publication.

Disclosures. Jens M. Hohlfeld's institution received funding from GlaxoSmithKline plc for study conduct. Jens M. Hohlfeld received honoraria for consulting and lectures from Boehringer Ingelheim, HAL Allergy, Merck and Novartis outside the scope of this work. David A. Hall, Kate Hanrott, David C. Budd, Rhena Eames, William M. Powley, Deborah Hewens, Aili L. Lazaar, Anthony Cahn and Sarah Siederer are employees of GlaxoSmithKline plc and own shares in the company. Philipp Badorrek and Dominik Berliner have nothing to disclose.

Compliance with Ethics Guidelines. All participants provided written informed consent, and the independent ethics committee of the Hannover Medical School reviewed and approved the study. The study was conducted in accordance with the Declaration of Helsinki, the International Council on Harmonisation, Good Clinical Practice and local regulations.

Data Availability. The datasets generated during and/or analysed during the current study are not publicly available because these data were used under license from Apeiron for the current study and restrictions apply to the availability of these data.

Open Access. This article is licensed under a Creative Commons Attribution-NonCommercial 4.0 International License, which permits any non-commercial use, sharing, adaptation, distribution and reproduction in any medium or format, as long as you give appropriate credit to the original author(s) and the source, provide a link to the Creative Commons licence, and indicate if changes were made. The images or other third party material in this article are included in the article's Creative Commons licence, unless indicated otherwise in a credit line to the material. If material is not included 
in the article's Creative Commons licence and your intended use is not permitted by statutory regulation or exceeds the permitted use, you will need to obtain permission directly from the copyright holder. To view a copy of this licence, visit http://creativecommons.org/licenses/by$\mathrm{nc} / 4.0 /$.

\section{REFERENCES}

1. Cargill RI, Lipworth BJ. Lisinopril attenuates acute hypoxic pulmonary vasoconstriction in humans. Chest. 1996;109:424-9.

2. Nong Z, Stassen JM, Moons L, Collen D, Janssens S. Inhibition of tissue angiotensin-converting enzyme with quinapril reduces hypoxic pulmonary hypertension and pulmonary vascular remodeling. Circulation. 1996;94:1941-7.

3. de Man FS, Tu L, Handoko ML, et al. Dysregulated renin-angiotensin-aldosterone system contributes to pulmonary arterial hypertension. Am J Respir Crit Care Med. 2012;186:780-9.

4. Camelo JS Jr, Hehre D, Devia C, Camelo SH, Bancalari E, Suguihara C. The role of angiotensin II receptor-1 blockade in the hypoxic pulmonary vasoconstriction response in newborn piglets. Neonatology. 2008;93:263-8.

5. Bader M. ACE2, angiotensin-(1-7), and Mas: the other side of the coin. Pflugers Arch. 2013;465: 79-85.

6. Shenoy V, Ferreira AJ, Qi Y, et al. The angiotensinconverting enzyme 2/angiogenesis-(1-7)/Mas axis confers cardiopulmonary protection against lung fibrosis and pulmonary hypertension. Am J Respir Crit Care Med. 2010;182:1065-72.

7. Zhang F, Tang H, Sun S, et al. Angiotensin-(1-7) induced vascular relaxation in spontaneously hypertensive rats. Nitric Oxide. 2019;88:1-9.

8. Sommer N, Ghofrani A, Pak O, et al. Current and future treatments of pulmonary arterial hypertension. Br J Pharmacol. 2020;178:6-30.

9. Paternot A, Repesse X, Vieillard-Baron A. Rationale and description of right ventricle-protective ventilation in ARDS. Respir Care. 2016;61:1391-6.

10. Kaisers U, Busch T, Deja M, Donaubauer B, Falke KJ. Selective pulmonary vasodilation in acute respiratory distress syndrome. Crit Care Med. 2003;31: S337-42.
11. Krebs MO, Boemke W, Simon S, Wenz M, Kaczmarczyk G. Acute hypoxic pulmonary vasoconstriction in conscious dogs decreases renin and is unaffected by losartan. J Appl Physiol. 1999;86: 1914-9.

12. Hubloue I, Rondelet B, Kerbaul F, et al. Endogenous angiotensin II in the regulation of hypoxic pulmonary vasoconstriction in anaesthetized dogs. Crit Care. 2004;8:R163-71.

13. Kiely DG, Cargill RI, Lipworth BJ. Effects of hypercapnia on hemodynamic, inotropic, lusitropic, and electrophysiologic indices in humans. Chest. 1996;109:1215-21.

14. Kleinsasser A, Pircher I, Treml B, et al. Recombinant angiotensin-converting enzyme 2 suppresses pulmonary vasoconstriction in acute hypoxia. Wilderness Environ Med. 2012;23:24-30.

15. Shen D, Wang Y. Changes of plasma level of neurotensin, somatostatin, and dynorphin A in pilots under acute hypoxia. Mil Med. 1998;163:120-1.

16. Grunig E, Mereles D, Hildebrandt W, et al. Stress Doppler echocardiography for identification of susceptibility to high altitude pulmonary edema. J Am Coll Cardiol. 2000;35:980-7.

17. Khan A, Benthin C, Zeno B, et al. A pilot clinical trial of recombinant human angiotensin-converting enzyme 2 in acute respiratory distress syndrome. Crit Care. 2017;21:234.

18. Naeije R, Saggar R, Badesch D, et al. Exercise-induced pulmonary hypertension: translating pathophysiological concepts into clinical practice. Chest. 2018;154:10-5.

19. Ricart A, Maristany J, Fort N, Leal C, Pages T, Viscor G. Effects of sildenafil on the human response to acute hypoxia and exercise. High Alt Med Biol. 2005;6:43-9.

20. Hemnes AR, Rathinasabapathy A, Austin EA, et al. A potential therapeutic role for angiotensin-converting enzyme 2 in human pulmonary arterial hypertension. Eur Respir J. 2018;2018:51.

21. Haschke M, Schuster M, Poglitsch M, et al. Pharmacokinetics and pharmacodynamics of recombinant human angiotensin-converting enzyme 2 in healthy human subjects. Clin Pharmacokinet. 2013;52:783-92.

22. Kiely DG, Cargill RI, Lipworth BJ. Acute hypoxic pulmonary vasoconstriction in man is attenuated by type I angiotensin II receptor blockade. Cardiovasc Res. 1995;30:875-80. 